\title{
Aurophilicity as a cofactor in crystal engineering. Dicyanoaurate(I) anion as a building block in a novel $\mathrm{Co}(\mathrm{II})-\mathrm{Au}(\mathrm{I})$ bimetallic assembly
}

\author{
Enrique Colacio, ${ }^{a}$ Francesc Lloret, ${ }^{b}$ Raikko Kivekäs, ${ }^{c}$ José Ruiz, $^{a}$ José Suárez-Varela ${ }^{a}$ and Markku R. \\ Sundberg $c$ \\ a Departamento de Química Inorgánica, Facultad de Ciencias, Universidad de Granada, 18071 Granada, \\ Spain.E-mail:ecolacio@ugr.es \\ ${ }^{b}$ Departamento de Química Inorgánica, Facultad de Química de la Universidad de Valencia, E-46100 \\ Burjassot, Valencia, Spain \\ c Department of Chemistry, Laboratory of Inorganic Chemistry, University of Helsinki, P.O. Box 55, \\ FIN-00014, Finland
}

Received (in Cambridge, UK) 5th December 2001, Accepted 5th February 2002

First published as an Advance Article on the web 20th February 2002

A 2D grid-shaped cyanide-bridged $\mathrm{Co}(\mathrm{II})-\mathrm{Au}(\mathrm{I})$ bimetallic coordination polymer, $\left[\mathrm{Co}(\mathrm{DMF})_{2}\left\{\mathrm{Au}(\mathrm{CN})_{2}\right\}_{2}\right]$, has been prepared from the $\left[\mathrm{Au}(\mathrm{CN})_{2}\right]^{-}$building block; sheets associate pair-wise by aurophilic interactions and the compound exhibits zeolite-like properties.

Cyanide-bridged bimetallic systems, prepared from assembling cyanometallates and transition metal complexes building blocks, have been shown to exhibit fascinating structures with interesting magnetic, electrochemical, magneto-optical and zeolitic properties. ${ }^{1}$ Linear cyanometallates, $\left[\mathrm{M}(\mathrm{CN})_{2}\right]^{-}(\mathrm{M}=$ $\operatorname{Ag}(\mathrm{I})$ and $\operatorname{Au}(\mathrm{I}))$, however, have been infrequently used as building blocks, probably as a consequence of their diamagnetic nature. Interestingly, gold(I) atoms of dicyanoaurate groups may be involved in intermolecular interactions comparable to hydrogen bonds. This closed-shell intermolecular interaction between gold(I) atoms, sometimes called aurophilicity, is known to be an useful tool for the design of intriguing and interesting polymeric structures in solid state. ${ }^{2}$ Therefore, the interplay between covalent and aurophilic interactions involving dicyanoaurate groups might produce novel structural topologies. Moreover, according to precise theoretical calculations, the $\mathrm{Au}-\mathrm{Au}$ interaction is effective over a wide range of distances. ${ }^{3}$ In a earlier statistical study ${ }^{4}$ based on 693 goldcontaining structures, it was found that there exists a strong correlation between the $\mathrm{Au}-\mathrm{Au}$ distance (in the range 3-4 $\AA$ ) and the dihedral angle between the donor atoms attached to the two adjacent gold atoms: the shorter the $\mathrm{Au}-\mathrm{Au}$ distance is, the higher will be the probability of the staggered conformation. This has been also observed for dicyanoaurate containing compounds. ${ }^{5}$

An interesting cation used in synthetic work with dicyanoaurate(I) anions is the cobalt(II) cation with its ability to display either octahedral or tetrahedral coordination. ${ }^{6}$ For both chromophores the resulting structure in the solid state is a threedimensional network, where both ends of the dicyanoaurate(I) anions are coordinated to a cobalt(II) cation. Interestingly, the shortest $\mathrm{Au}-\mathrm{Au}$ distances are significantly different: $3.11 \AA$ in the tetrahedral and $3.33 \AA$ in the octahedral analogue. If some of the coordination sites of a cation are blocked by an additional ligand, the dimensionality can be varied and then it might be possible to analyse the influence of the aurophilic interactions on the final structure. For instance, in a formally 1D structure of (tmeda) $\mathrm{Cu}\left[\mathrm{Au}(\mathrm{CN})_{2}\right]_{2}$ (tmeda $=N, N, N^{\prime}, N^{\prime}$-tetramethylethylenediamine $)^{7}$ the aurophilicity enhances the dimensionality to $3 \mathrm{D}$, with $\mathrm{Au}-\mathrm{Au}$ distances of 3.345 and $3.538 \AA$, less than the sum of the van der Waals radii of $A u(3.60 \AA)$.

Here we applied the same strategy in synthetic work. ${ }^{8}$ From the reaction of $\mathrm{K}\left[\mathrm{Au}(\mathrm{CN})_{2}\right]$ and $\mathrm{Co}(\mathrm{Ac})_{2} \cdot 4 \mathrm{H}_{2} \mathrm{O}$ in DMF the bimetallic assembled compound $\left[\mathrm{Co}(\mathrm{DMF})_{2}\left\{\mathrm{Au}(\mathrm{CN})_{2}\right\}_{2}\right](\mathbf{1})$ was obtained as pink crystals. The crystal structure of $\mathbf{1}^{9}$ consists of parallel sheets lying in $b c$-plane, made up of edgesharing approximate square-planar $\{\mathrm{NC}-\mathrm{Au}-\mathrm{CN}-\mathrm{Co}\}_{4}$ units with $\mathrm{Co}$ (II) ions and bridging $\left[\mathrm{Au}(\mathrm{CN})_{2}\right]^{-}$anions at each corner and edge, respectively. A perspective view of the asymmetric unit together with a section of the 2D grid-shaped structure of 1 are shown in Fig. 1.

Each cobalt assumes a slightly distorted octahedral $\mathrm{CoN}_{4} \mathrm{O}_{2}$ coordination polyhedron, which is made up of four nitrogen atoms from the bridging cyanide groups in plane and two oxygen atoms of the DMF molecules in trans-positions. The $\mathrm{Co}-\mathrm{N}$ bond distances are in the range 2.086(11) to 2.116(12) $\AA$ whereas Co-O bond distances are 2.088(10) and 2.093(10) A. As expected, the two crystallographically non-equivalent $\left[\mathrm{Au}(\mathrm{CN})_{2}\right]^{-}$anions are almost linear with $\mathrm{C}-\mathrm{Au}-\mathrm{C}$ bond angles of 177.9(6) and $175.5(6)^{\circ}$. The $\mathrm{CN}-\mathrm{Co}$ and $\mathrm{NC}-\mathrm{Au}$ bond angles do not deviate much from linearity with values in the ranges 168.6(11)-173.5(11) and 173.8(12)-177.8(13) ${ }^{\circ}$, respectively. Within each square unit of a layer the Co(II)-Co(II) distances are 14.054(5) and 10.304(3) ̊ for atoms lying diagonally opposite and for atoms joined by $\left[\mathrm{Au}(\mathrm{CN})_{2}\right]^{-}$bridging groups, respectively. There are inversion centres lying at the centres of the square units, so that the $\mathrm{CoN}_{4}$ planes of diagonally opposite $\mathrm{Co}$ (II) atoms are parallel, whereas the angle between planes of neighbouring $\mathrm{Co}$ (II) atoms bridged by $\left[\mathrm{Au}(\mathrm{CN})_{2}\right]^{-}$is $24.0(2)^{\circ}$. Sheets are then not planar but slightly corrugated. Each sheet is shifted with respect the next-neighbouring ones so that the cobalt(II) atoms of one sheet lie above and below the centres of the square units of the neighbouring sheets, giving rise to a $\mathrm{ABAB} . .$. sequence pattern. In this disposition part of the DMF molecules can interpenetrate into the holes of square-planar $\{\mathrm{NC}-\mathrm{Au}-\mathrm{CN}-\mathrm{Co}\}_{4}$ units. This relieves steric crowding and allows interlayer aurophilic interactions between two adjacent planes with $\mathrm{Au}-\mathrm{Au}$ distances of 3.1949(10) $\AA$ that is shorter than the sum of their van der Waals radii. These gold(I)-gold(I) bonding interactions lead to a very short average interlayer separation of $3.12 \AA$. Structurally, the coordinated DMF molecules have double characteristics: within the pair-wise associated sheets, part of them are filling the holes of the neighbouring $\{\mathrm{NC}-\mathrm{Au}-\mathrm{CN}-\mathrm{Co}\}_{4}$ units, as mentioned above,

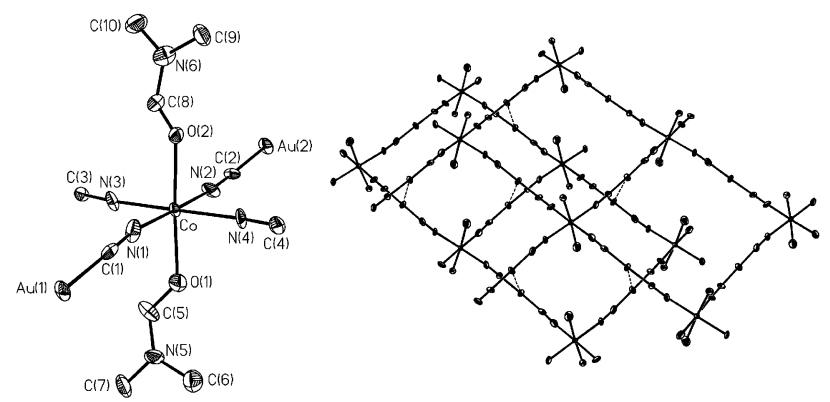

Fig. 1 Perspective views of the asymmetric unit and two pair-wise associated sheets (in the latter, and for the sake of clarity, only the $\mathrm{O}(2)$ atom of the DMF ligand is included) with $50 \%$ probability ellipsoids. 
and another part of them is oriented away from the pair resulting in a rather long distance $(5.25 \AA)$ between neighbouring pairs of sheets. Noteworthy, in $\left[\mathrm{Co}(\mathrm{pz})_{2}(\mathrm{NCS})_{2}\right],{ }^{10}$ containing bis(monodentate) pyrazine bridging ligands between $\mathrm{Co}(\mathrm{II})$ atoms and thiocyanate ligands in axial positions of the Co(II) atoms, the interlayer separation $(8.487 \AA$ ) is much longer than that for $\mathbf{1}$, which is clearly due to the absence of aurophilic interactions.

It should be noted that, as far as we know, there is only one previous computational study ${ }^{11}$ on dicyanoaurates, where extended Hückel calculations augmented by relativistic parameters were carried out. Now, and in order to theoretically study the influence of the charge and oligomerisation state of the dicyanoaurate units involved in aurophilic interactions on the observed $\mathrm{C}-\mathrm{Au}-\mathrm{Au}^{\prime}-\mathrm{C}^{\prime}$ torsion angle and other structural factors, we have performed structure optimisations at the MP2/ LANL2DZ level of theory by making use of the Gaussian 98 code. Where appropriate, the initial $\mathrm{C}-\mathrm{Au}-\mathrm{Au}^{\prime}-\mathrm{C}^{\prime}$ torsion angles were $45^{\circ}$ and the $\mathrm{Au}-\mathrm{Au}$ ' distances were $3.3 \AA$. No symmetry restrictions were applied during the optimisations. According to the calculated IR spectra all the optimised structures represent true energy minima. Selected geometrical parameters for the optimised moieties are shown in Table 1.

Table 1 Selected geometrical parameters for the optimised moieties

\begin{tabular}{lllr}
\hline Moiety & $\mathrm{Au}-\mathrm{Au} / \AA$ & $\mathrm{C}-\mathrm{Au}-\mathrm{C} /{ }^{\circ}$ & $\mathrm{C}-\mathrm{Au}-\mathrm{Au}^{\prime}-\mathrm{C}^{\prime} /^{\circ}$ \\
\hline Monomer anion & & 180.0 & \\
Dimer anion & 3.472 & 173.6 & 90 \\
Neutral dimer & 3.893 & 170.1 & 0 \\
Mixed trimer & 3.180 & 178.6 & 90 \\
Trimer anion & 3.180 & 180.0 & 90 \\
\hline
\end{tabular}

The charge of the moieties obviously has several consequences. First of all, the torsion angle $\mathrm{C}-\mathrm{Au}-\mathrm{Au}^{\prime}-\mathrm{C}^{\prime}$ changes considerably, since in the start of every optimisation the torsion angle was $45^{\circ}$. In the neutral dimer the torsion angle is $0^{\circ}$, whereas in the anionic dimer it is $90^{\circ}$. In the neutral dimeric moiety the $\mathrm{Au} \cdots \mathrm{Au}$ ' distance of $3.893 \AA$ is longer than the sum of the van der Waals radii, confirming the absence of aurophilicity, which is also corroborated of the torsion angle of $0^{\circ}$. Also the angle $\mathrm{C}-\mathrm{Au}-\mathrm{C}$ varies; especially for the anionic dimer the deformation can be taken as evidence for a gold(I)gold(I) interaction. This is illustrated in Fig. 2.

A similar torsion angle of $90^{\circ}$ is also seen in the trimeric moiety, where the anionic form is in the middle. These results agree well with the observed staggered conformation for dicyanoaurate anions involved in aurophilic interactions.

As for the magnetic properties, the $\chi_{\mathrm{M}} T$ product at room temperature per cobalt(II) atom of $3.03 \mathrm{~cm}^{3} \mathrm{~mol}^{-1} \mathrm{~K}$ decreases upon cooling until reaching a value of $1.8 \mathrm{~cm}^{3} \mathrm{~mol}^{-1} \mathrm{~K}$ at $2 \mathrm{~K}$. To account for the experimental results it is necessary to consider spin-orbit coupling, electron delocalization and a lowsymmetry ligand field component. Thus, the magnetic behaviour of 1 can be theoretically modelled as a monomeric tetragonally distorted octahedral cobalt(II) complex with $k=$ 0.8 ( $k$ is the orbital reduction parameter) and $\delta=1200 \mathrm{~cm}^{-1}(\delta$
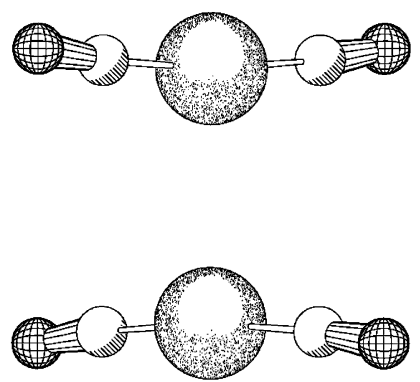

Fig. 2 PLUTON illustration of the dimeric dianion optimized at the MP2/ LANL2DZ level of theory. is the splitting of the ${ }^{4} \mathrm{~T}_{1 \mathrm{~g}}$ term by tetragonal distortion), and fixed values of $\lambda=170 \mathrm{~cm}^{-1}$ ( $\lambda$ is the spin-orbit coupling parameter) and $A=1.35$ (medium to weak ligand field). This clearly indicates that the magnetic exchange interaction between cobalt(II) ions mediated by dicyanoaurates groups, if exists, is negligible.

Finally, the thermogravimetric study of $\mathbf{1}$, under an oxygen atmosphere and combined with an FT-IR analysis of the gases, shows that both DMF molecules are released in one step in the 200-250 ${ }^{\circ} \mathrm{C}$ temperature range (weight loss: calc., 20.76\%; found, $20.26 \%$ ). It is of note that when the heated compound is allowed to stand at room temperature it absorbs water from the ambient moisture leading to $\left[\mathrm{Co}\left(\mathrm{H}_{2} \mathrm{O}\right)_{2}\left\{\mathrm{Au}(\mathrm{CN})_{2}\right\}_{2}\right]$. This complex loses these coordinated water molecules in the temperature range $120-180{ }^{\circ} \mathrm{C}$ (calc., $6.07 \%$; found, $6.30 \%$ ). If the structure of $\mathbf{1}$ is maintained when the solvent is lost, and because the process is reversible, the heated compound would exhibit sieving properties resembling zeolites. A deeper study of the zeolitic properties of $\mathbf{1}$ and analogous materials is in progress. The results for $\mathbf{1}$ represent new evidence that solid materials that can reversibly absorb small molecules do not need to be porous. ${ }^{12}$

In conclusion, when the dicyanoaurate anion is used as a building block, the interplay between covalent and aurophilic bonding interactions has been shown to be a appropriate approach to prepare bimetallic $\mathrm{M}-\mathrm{Au}(\mathrm{I})$ compounds exhibiting interesting structures and physical properties. Reactions between $\left[\mathrm{M}(\mathrm{CN})_{2}\right]^{-}$and a wide variety of transition metal complexes are currently under examination.

This work was supported by the Dirección General de Enseñanza Superior (project PB97-0822) and the Academy of Finland (project 41519, RK).

\section{Notes and references}

1 See, for example: K. R. Dumbar and R. A. Heintz, Prog. Inorg. Chem., 1997, 45, 283; M. Verdaguer, A. Bleuzen, V. Marvaud, J. Vaissermann, M. Seuleiman, C. Desplanches, A. Scuiller, C. Train, R. Garde, G. Gelly, C. Lomenech, I. Rosenman, P. Veillet, C. Cartier and F. Villain, Coord. Chem. Rev., 1999, 190, 1023; M. Ohba and K. Okawa, Coord. Chem. Rev., 2000, 198, 313.

2 H. Schmidbaur, Chem. Soc. Rev., 1995, 24, 391; P. Pyykkö, Chem. Rev., 1997, 97, 597.

3 P. Pyykkö and N. Runeberg, Chem. Phys. Lett., 1994, 218, 133.

4 S. S. Pathaneni and G. R. Desiraju, J. Chem. Soc., Dalton Trans., 1993 319.

5 R. E. Cramer, D. W. Smith and W. Vandoorne, Inorg. Chem., 1998, 37, 5895 .

6 S. C. Abrahams, J. L. Bernstein and R. Liminga, J. Chem. Phys., 1980 73, 4585; S. C. Abrahams, L. E. Zyontz and J. L. Bernstein, J. Chem. Phys., 1982, 76, 5458

7 D. B. Leznoff, B.-Y. Xue, B. O. Patrick, V. Sanchez and R. C. Thompson, Chem. Commun., 2001, 259.

8 Synthesis of 1: A $50 \mathrm{ml}$ water-acetone $(1: 4)$ solution of $\mathrm{K}\left[\mathrm{Au}(\mathrm{CN})_{2}\right]$ $(0.1 \mathrm{~g}, 0.35 \mathrm{mmol})$ was added to an aqueous solution of $\mathrm{Co}(\mathrm{Ac})_{2} \cdot 4 \mathrm{H}_{2} \mathrm{O}$ $(0.043 \mathrm{~g}, 0.17 \mathrm{mmol})$. The resulting pink solution kept at room temperature for several days afforded a pink precipitate, which was filtered off, washed with water and air-dried. Slow evaporation of a solution of this precipitate in DMF gave pink prismatic crystals. Yield $25 \%$ based on Co. Anal. Calc. for $\mathrm{C}_{10} \mathrm{H}_{14} \mathrm{~N}_{6} \mathrm{O}_{2} \mathrm{Au}_{2} \mathrm{Co}$ : C, 17.06; $\mathrm{H}$, 1.99 ; N, 11.95. Found: C, 17.22; H, 2.05; N, 11.72\%. IR(KBr): $v_{\mathrm{CN}}$ $2179, v_{\mathrm{CO}} 1664 \mathrm{~cm}^{-1}$

9 Crystal data: $\mathrm{C}_{10} \mathrm{H}_{14} \mathrm{~N}_{6} \mathrm{O}_{2} \mathrm{Au}_{2} \mathrm{Co}$; monoclinic, space group $P 2{ }_{1} / c, a=$ $8.375(2), b=14.054(5), c=15.077(4) \AA, \beta=92.75(2)^{\circ}, U=$ 1772.6(9) $\AA^{3}, Z=4, D_{\mathrm{c}}=2.635 \mathrm{~g} \mathrm{~cm}^{-3}, \mu(\mathrm{Mo}-\mathrm{K} \alpha)=17.447 \mathrm{~mm}^{-1}$, $T=193 \mathrm{~K} . F(000)=1268.3189$ reflections were collected by $\omega / 2 \theta$ scans $\left(2 \theta_{\max }=50^{\circ}\right)$. The structure was solved by direct methods and refined on $F^{2}$ by the SHELX-97 program to $R 1=0.0563(w R 2=$ 0.1422 ) with a goodness-of-fit of 0.992 . CCDC reference number 173686. See http://www.rsc.org/suppdata/cc/b1/b110020c/ for crystallographic data in CIF or other electronic format.

10 F. Lloret, G. DeMunno, M. Julve, J. Cano, R. Ruiz and A. Caneschi, Angew. Chem., Int. Ed., 1998, 37, 135.

11 M. A. Rawadesh-Omary, M. A. Omary and H. H. Patterson, J. Am Chem. Soc., 2000, 122, 10371.

12 M. Albrecht, M. Lutz, A. L. Speek and G. van Koten, Nature, 2000, 406, 970 . 